\title{
Unraveling the Impact of High-Order Silk Structures on Molecular Drug Binding and Release Behaviors
}

\author{
Thidarat Wongpinyochit, ${ }^{\dagger}$ Antony D. Vassileiou, ${ }^{\dagger}$ (๑) Sukriti Gupta, ${ }^{\ddagger},{ }^{\S}$ Samir H. Mushrif, ${ }^{\S}, \|_{\odot}$ \\ Blair F. Johnston, $*^{\dagger},, \perp_{(}$and F. Philipp Seib ${ }^{*},, \# \mathbb{C}$
}

\begin{abstract}
${ }^{\dagger}$ Strathclyde Institute of Pharmacy and Biomedical Sciences, University of Strathclyde, 161 Cathedral Street, Glasgow G4 0RE, United Kingdom

${ }^{\ddagger}$ Energy Research Institute @ NTU, Interdisciplinary Graduate School, Nanyang Technological University, 50 Nanyang Drive, Singapore 637553

${ }^{\S}$ School of Chemical and Biomedical Engineering, Nanyang Technological University, 62 Nanyang Drive, Singapore 637459

"Department of Chemical and Materials Engineering, University of Alberta, 9211-116 Street Northwest, Edmonton, Alberta T6G 1H9, Canada

${ }^{\perp}$ National Physical Laboratory, Teddington, Middlesex TW11 0LW, United Kingdom

\#Leibniz Institute of Polymer Research Dresden, Max Bergmann Center of Biomaterials Dresden, Hohe Strasse 6, 01069 Dresden, Germany
\end{abstract}

Supporting Information

ABSTRACT: Silk continues to amaze: over the past decade, new research threads have emerged that include the use of silk fibroin for advanced pharmaceutics, including its suitability for drug delivery. Despite this ongoing interest, the details of silk fibroin structures and their subsequent drug interactions at the molecular level remain elusive, primarily because of the difficulties encountered in modeling the silk fibroin molecule. Here, we generated an atomistic silk model containing amorphous and crystalline regions. We then exploited advanced well-tempered metadynamics simulations to generate molecular conformations that we subsequently exposed to classical molecular dynamics simulations to monitor both drug binding and release. Overall, this study demonstrated the importance of the silk fibroin primary

sequence, electrostatic interactions, hydrogen bonding, and higher-order conformation in the processes of drug binding and release.
F or millennia, humans have used silk thread ${ }^{1}$ as a suture material. ${ }^{2,3}$ However, the use of the silk fibroin protein for drug delivery applications, including its use as a nanomedicine for lysosomotropic drug delivery, ${ }^{4}$ has only recently been reported. ${ }^{5,6}$ The primary and higher-order structures of the silk fibroin protein are fundamental for its function as a high-tech fiber, but they also dictate the ability of silk fibroin to serve as a biopolymer for drug delivery. ${ }^{5,6}$ Clinically relevant therapeutic agents have been successfully loaded into silk films, ${ }^{7,8}$ hydrogels, ${ }^{9,10}$ and nanoparticles ${ }^{4,11,12}$ (reviewed in ref 13), with an increasing number of studies now specifically reporting the use of recombinant silks ${ }^{14}$ and silk-elastin-like proteins for anticancer drug delivery (e.g., doxorubicin as a payload). ${ }^{10,12}$ However, the precise mechanism that controls the interaction between the payload and silk at the atomic level is not fully known at present, although seminal studies by Coburn and coworkers have demonstrated the importance of charge and crystallinity on drug binding and release. ${ }^{8}$ Molecular dynamics simulations are now recognized as a powerful tool for

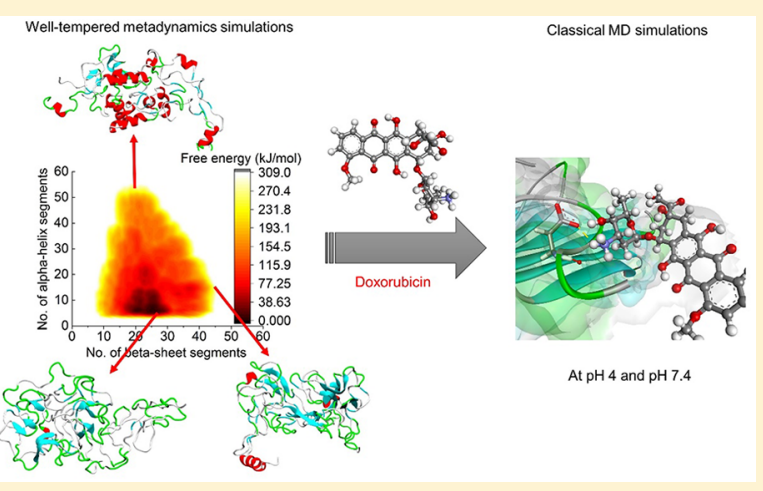
demonstrating the binding mechanism between a drug (e.g., doxorubicin) and several types of drug carrier molecules, such as nanodiamond, ${ }^{15}$ graphene oxides, ${ }^{16}$ and chitosan oligosaccharide. ${ }^{17}$ Currently, van der Waals forces, electrostatic interactions, and hydrogen bonding are suggested as the key factors that enable doxorubicin binding to a carrier.

Molecular dynamics simulations have been widely used to investigate the motion of large molecules such as proteins (e.g., protein folding and ligand binding at the nanoscale). ${ }^{18,19}$ For more than a decade, molecular dynamics simulation has been used to study silk structures, their mechanical properties, ${ }^{20-22}$ and the interactions between silk and other molecules (e.g., graphene). ${ }^{23}$ However, many silk structures used previously were small (e.g., a stack of poly(GA) $\beta$-sheets $<100$ amino acids long), ${ }^{22,24,25}$ and the amino acid composition did not

Received: June 3, 2019

Accepted: July 9, 2019 
A

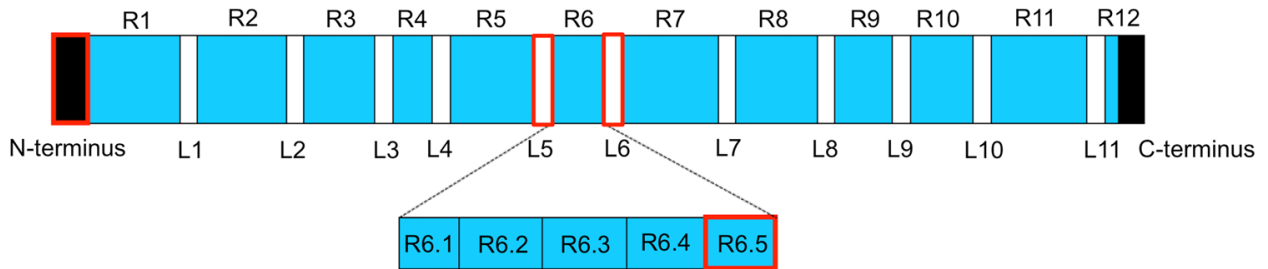

\section{B Amorphous-crystalline domain}

\begin{tabular}{|l|l|l|}
\hline \multicolumn{1}{|c|}{ L5 } & $\mathrm{R} 6.5$ & $\mathrm{~L} 6$ \\
\hline L6 & $\mathrm{R} 6.5$ & $\mathrm{~L}$ \\
\hline
\end{tabular}

Chain A abGSSGFGPYVAHGGYSGYEYAWSSESDFGTGScaGScccGAGSccdcGAASabGSSGFGPYVANGGYSGYEYAWSSESDFGTGS

Chain B SGTGFDSESSWAYEYGSYGGNAVYPGFGSSGbaSAAGcdccSGAGcccSGacSGTGFDSESSWAYEYGSYGGNAVYPGFGSSGba

$\mathrm{a}=\mathrm{GAGAGA}, \mathrm{b}=$ GAGAGT, $\mathrm{c}=$ GAGAGS, $\mathrm{d}=$ GAGAGY

\section{N-terminal domain}

\section{Chain A FDEDYFGSDVTVQSSNTTDEIIRDASGAVIEEQITTKKMQRKNILGKNEKMIKTFVITTDSDGNESIVEEDVLMKTLSD}

Chain B INDFDEVTVQSSNTTDEIIRDASGAVIEEQITTKKMQRNEKMIKTFVITTDSDGNESIVEEDVLMKTLSD

Figure 1. Primary structure of the Bombyx mori silk fibroin heavy chain and the amino acid sequences used for molecular dynamics simulations. (A) Schematic representation of the primary structure of the B. mori silk fibroin heavy chain. R1-R12 represent 12 repeated crystalline domains, and L1-L11 represent 11 linkers (amorphous regions). The subdomains L5, R6.5, and L6 and the N-terminus (red squares) are used in this study. The subdomain sequences are available on Uniprot databases in a FASTA file named P05790 at position number 2281-2323 (L5), 2536-2599 (R6.5), and 2600-2642 (L6). (B) Schematic representation of the antiparallel silk structures (arrows show the directions) and their amino acid sequences. (C) Amino acid sequence of the $\mathrm{N}$-terminal domain (available in the Protein Data Bank [3UA0]). ${ }^{31}$

faithfully reflect key components of the silk structure. A key result of the present study has been the generation and modeling of silk fibroin fragments totaling 300 amino acids in length and containing both amorphous (linkers) and crystalline domains (Figure 1A,B). This fragment is a good representation of the whole silk structure because the R6.5 subdomain contains the most frequently repeating crystalline sequences of poly(GA) and GAGAS, flanked by linker 5 and linker 6 (ref 26); these silk elements have been used by others and thus provide a useful reference point. ${ }^{23}$ The use of our 300 amino acid long structure for molecular dynamics simulation is challenging because of the time scales required for full exploration of the conformation space and because of the conformational energy barriers. ${ }^{27}$ This challenge can be addressed by using advanced sampling algorithms, such as well-tempered metadynamics. ${ }^{28,29}$ The well-tempered metadynamics method improves the configurational sampling during molecular dynamics simulations by adding an external historydependent potential, along with selected collective variables that enable enhanced sampling and reconstruction of free energy surfaces. ${ }^{29,30}$ Here, we used well-tempered metadynamics to explore the conformational free energy profiles of amorphous-crystalline silk structures in water. The collective variables were the number of $\beta$-sheet and $\alpha$-helix segments, which are key secondary structure components of silk (see the Supporting Information for a complete description of the modeling approaches employed). The simulation provided realistic three-dimensional amorphous-crystalline silk fibroin structures, which were then compared to known structures derived from NMR measurements. The silk structures were then selected to investigate silk-doxorubicin interactions in response to $\mathrm{pH}$ using classical molecular dynamics simulations. These data were compared to previously reported experimental studies.

The critical step for the well-tempered metadynamics simulations was the selection of the collective variables as these should be able to distinguish between relevant metastable states and to describe the key features of the structure. ${ }^{32}$ Thus, in this study, the number of $\beta$-sheets and $\alpha$-helix segments were the collective variables because the secondary structure of silk contains primarily these two structures. These collective variables can be detected and tracked to assess the conformational changes of the silk structure in water along their trajectories. The convergence of the system was monitored by reconstructing the time evolution profiles of the collective variables, the Gaussian height, and the free energy landscapes. At the convergence, three main questions needed to be considered: Are the collective variable values fully explored over the simulation time? Does the Gaussian height reach zero? Are the reconstructed free energy profiles similar? ${ }^{29}$ In this study, the well-tempered metadynamics simulations of the silk fibroin structure in water were converged after a $600 \mathrm{~ns}$ simulation because two collective variable values effectively diffused over the simulation (Figure S1A). Specifically, the height of the biased potential reached zero (Figure S1B), and no significant change occurred in the free energy surface shape over the last $50 \mathrm{~ns}$ (Figure S2). 

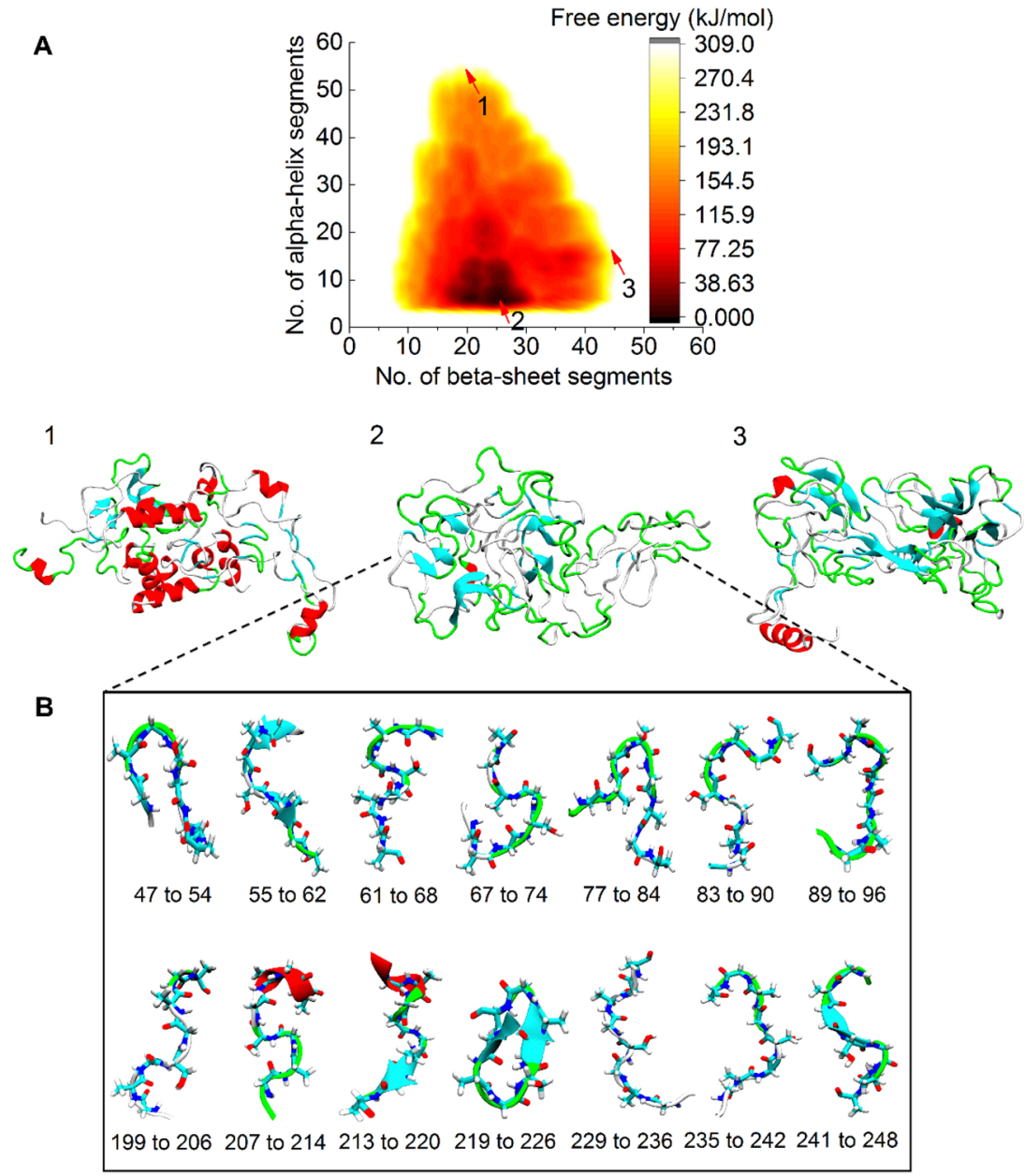

C

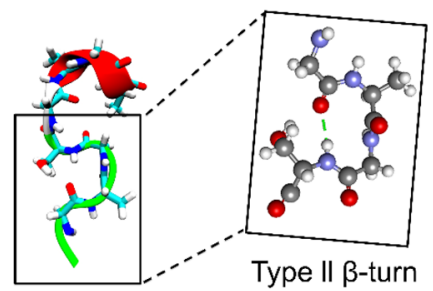

Figure 2. Converged free energy surface of the silk structure in water, showing the key structures and 14 repetitive sequence motifs (GAGSGAGA) in the crystalline domains of silk fibroin structure. (A) Free energy profile with three relevant silk structures: (1) the silk structure containing the highest number of $\alpha$-helix segments, (2) the most stable silk structure at the global minimum, and (3) the silk structure containing the highest number of $\beta$-sheet segments. (B) Fourteen repetitive sequence motifs (GAGSGAGA) extracted from the most stable structure after a $600 \mathrm{~ns}$ welltempered metadynamics simulation. The number is the residue number for the GAGSGAGA motif in the silk structure. (C) Repetitive sequence motif GAGSGAGA (at residue numbers 207-214) of the silk structure and its first four residues (GAGS) representing a type II $\beta$-turn structure with a hydrogen bond (green dashed line). The silk structures were colored using the Visual Molecular Dynamics software package: $\alpha$-helix $=$ red, $\beta$-sheet $=$ cyan, turn $=$ green, random-coil $=$ white

Next, the most interesting silk structures were extracted from the free energy surface at $600 \mathrm{~ns}$, for example, the most stable structure at the global minimum (Figure $2 \mathrm{~A}$ point 2 ), as well as the relevant structures containing either the highest number of $\alpha$-helix segments or the highest number of $\beta$-sheet segments (Figure $2 \mathrm{~A}$ points 1 and 3 , respectively). Silk I*, the ordered regions of silk I, represents the structure of the silk solution present in the middle silk gland. It is described as a type II $\beta$-turn structure and is the nucleus for silk II formation. ${ }^{33}$ Experimental evidence indicates that $55-58 \%$ of the GAGSGAGA sequence in silk I adopts the Silk I* structure, while the rest forms a random coil. ${ }^{33}$ To validate the simulation, the 14 repetitive GAGSGAGA sequences from the crystalline domains were extracted from the most stable silk structure (Figure 2B) and analyzed by (i) plotting the torsion angles phi and psi on a Ramachandran plot and (ii) examining the type II $\beta$-turn structure patterns (Figure S3).

NMR data have been reported for soluble silk (i.e., silk stored in the silkworm middle gland Figure S3B), and the torsion angles of silk structure were plotted (Figure S3A), adopting a type II $\beta$-turn structure model (Figure S3C). ${ }^{34}$ These literature torsion angles were then compared to the angles obtained from the silk structure after well-tempered metadynamics simulations. Here, $38 \%$ of the GAGSGAGA 


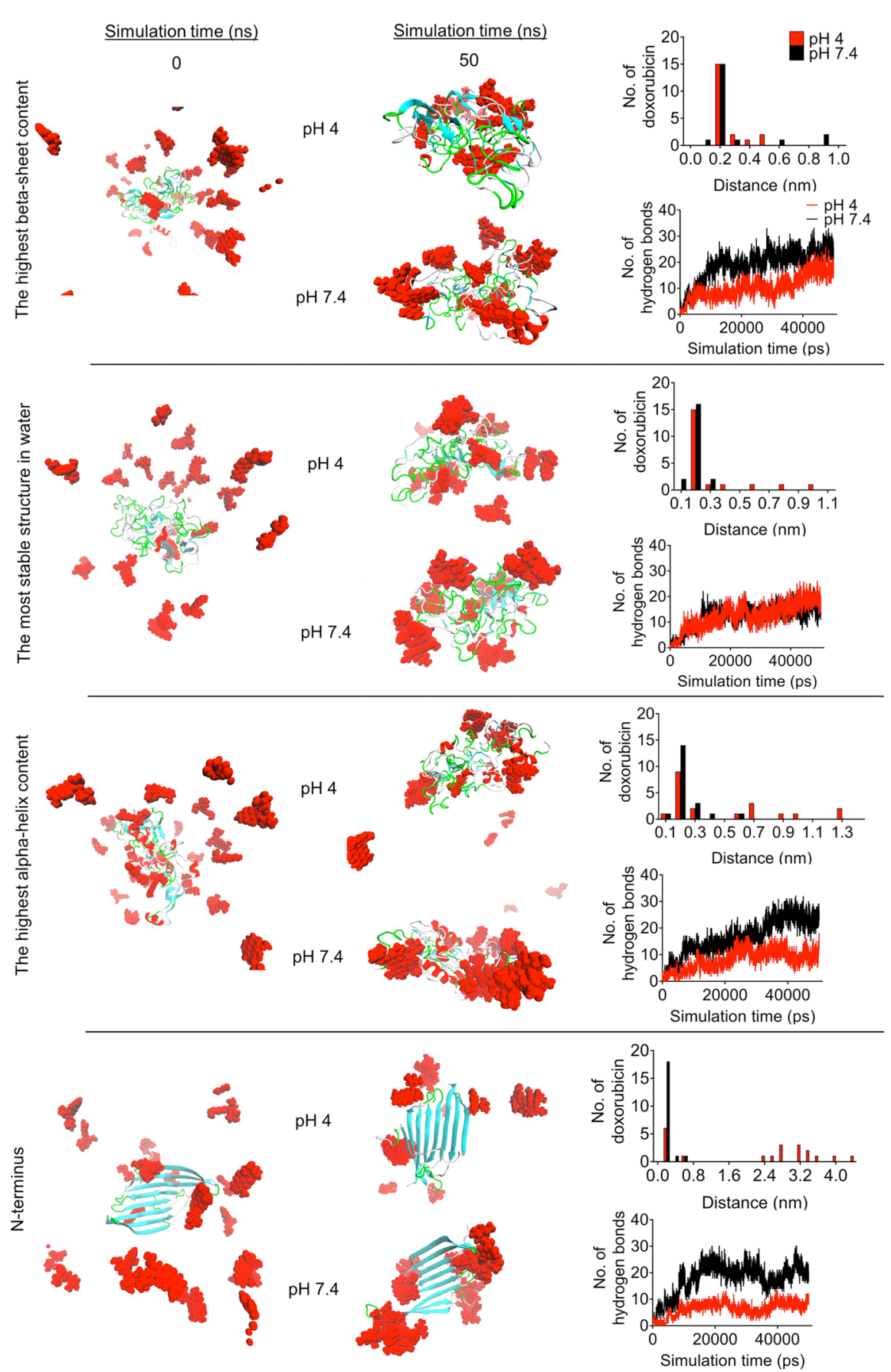

Figure 3. Snapshots of doxorubicin distribution on the silk fibroin structure at 0 and $50 \mathrm{~ns}$ in the molecular dynamics simulations at the indicated $\mathrm{pH}$ values of 4 and 7.4. The postprocessing analysis of the silk-doxorubicin system is presented as the distribution of the number of doxorubicin molecules at different distances ( $\mathrm{nm}$ ) from silk fibroin structures at $50 \mathrm{~ns}$ and the number of hydrogen bonds of all of the silk-doxorubicin systems over a $50 \mathrm{~ns}$ simulation time. The doxorubicin and silk fibroin structures were visualized using the Visual Molecular Dynamics software package; $\alpha$ helix $=$ red, $\beta$-sheet $=$ cyan, turn $=$ green, random-coil $=$ white, and doxorubicin $=$ red molecules .

from the respective sequence was aligned in the area of ideal torsion angles (red circles) (Figure S3A). All angles of the GAGSGAGA from residue numbers 207-214 were located within this area. These data were corroborated by analyzing the hydrogen bonds and the structural pattern using the Visual Molecular Dynamics software package. ${ }^{35}$ This analysis revealed a type II $\beta$-turn structure with one hydrogen bond between the $\mathrm{C}=\mathrm{O}$ of the glycine $(\mathrm{i})$ and the $\mathrm{H}-\mathrm{N}$ of the serine $(\mathrm{i}+3)^{33}$ (Figure 2C). Therefore, the silk model described in this study supports the current Silk I* conformation (i.e., the type II $\beta$ - turn structure) previously proposed based on NMR data. ${ }^{34}$ In addition, the modeling yielded some residues that formed unstructured segments not described previously.

Our previous work ${ }^{4,11,36}$ detailed the manufacture of silk nanoparticles, which were examined for drug loading and release, as well as for their capacity for internalization and trafficking in human breast cancer cells. These studies showed that doxorubicin loading onto silk nanoparticles could be accomplished by simple adsorption from a doxorubicin solution. In addition, drug release was $\mathrm{pH}$-dependent, with 
the fastest release occurring at an acidic $\mathrm{pH}$ (similar to the lysosomal $\mathrm{pH}$ of approximately 4.5). ${ }^{11,36}$ Dedicated wet-lab studies by Coburn and co-workers uncovered the importance of charge and crystallinity on drug binding and release. ${ }^{8}$ The best doxorubicin binding capacity was observed for lowcrystalline silk fibroin, and binding could be further increased by chemical modifications of the fibroin. By contrast, drug release depended on the protonation state of the silk fibroin, so that the fastest release occurred at a low $\mathrm{pH}^{8}{ }^{8}$ The present in silico study provides unprecedented atomistic insight into the mechanism underlying doxorubicin interaction with silk fibroin and the subsequent effects of $\mathrm{pH}$ on drug release. We therefore used the silk fibroin structures extracted from the welltempered metadynamics simulations to study the $\mathrm{pH}$-dependent interaction of doxorubicin with silk. The selected silk fibroin structures were (i) the most stable silk structure, (ii) the silk with the highest $\alpha$-helix content (i.e., Silk I), and (iii) silk with the highest $\beta$-sheet content (i.e., Silk II dominating silk nanoparticles). The $\mathrm{N}$-terminal domain structure (Nterminal sequence shown in Figure 1C) was also included because it contains a $\mathrm{pH}$-responsive region required for silk self-assembly. ${ }^{31}$

To mimic the experimental studies, the silk structure models were subjected to $\mathrm{pH} 7.4$ and 4 (for the experimental approach, see the SI), which are values that mimic blood plasma (as well as the loading solution) and the lysosome interior, respectively. The silk structure models used in this study contained five ionizable amino acids. The most important amino acids were aspartic acid, glutamic acid, and tyrosine in the amorphous-crystalline domain, whereas the $\mathrm{N}$ terminal domain contained aspartic acid, glutamic acid, tyrosine, lysine, and arginine (Figure $S 4$ ). The $\mathrm{p} K_{\mathrm{a}}$ of ionizable residues can be shifted in response to changes in the surrounding environment (e.g., solvent, $\mathrm{pH}$, etc.), which will change the protein conformation (e.g., hiding/exposing ionizable groups in the structure etc.). ${ }^{37}$ Therefore, the initial step in the simulation was to calculate the $\mathrm{p} K_{\mathrm{a}}$ of the ionizable amino acids in the silk models to allow modification of the protonation states at $\mathrm{pH} 4$ and 7.4 using Discovery Studio software.

The calculated $p K_{\mathrm{a}}$ values for ionizable residues were then used to estimate the total net charges at $\mathrm{pH} 4$ and 7.4 (Tables S1-S4). For the amorphous-crystalline domain of the model silk fibroin structure, the total net charge at $\mathrm{pH} 4$ was less negative than the charge at $\mathrm{pH} 7.4(-2$ to -4 at $\mathrm{pH} 4$ and -12 at $\mathrm{pH}$ 7.4). By contrast, the $\mathrm{N}$-terminal domain of the silk fibroin model contained a large number of ionizable amino acids; therefore, the total net charges of the $\mathrm{N}$-terminal domain at $\mathrm{pH} 4$ and 7.4 differed significantly $(+6$ at $\mathrm{pH} 4$ and -17 at $\mathrm{pH}$ 7.4) (Table S4). Across all structures, glutamic acid contributed substantially to the total net charge of all of the silk fibroin structure models studied here.

The differences in the total net charges of the silk fibroin structures can be visualized by generating the distribution of charged amino acid residues on the surfaces (Figure S5). The proportion of negatively charged amino acids was higher in the silk fibroin structure at $\mathrm{pH} 7.4$ than that at $\mathrm{pH}$ 4. Extra negative charges were evident for the amorphous-crystalline silk structure at $\mathrm{pH} 7.4$ due to the presence of glutamic acid (residue number 37, 290, 296) and aspartic acid (residue number 39) (Figure S5A). For the N-terminal domain, the surface charge distribution at $\mathrm{pH} 4$ and 7.4 was already visually different (Figure S5B) due to the high number of positive charges at $\mathrm{pH} 4(+6)$ and the high number of negative charges at $\mathrm{pH} 7.4(-17)$ (Table S4).

The model system consisted of modified protonation states of the model silk fibroin structure (four separate model systems), 20 doxorubicin molecules, and water. The molecular dynamics simulations of all systems were run for $50 \mathrm{~ns}$ in GROMACS packages. We first analyzed the spatial distribution of 20 doxorubicin molecules around the silk fibroin model structures and specifically analyzed the doxorubicin position at the end of the $50 \mathrm{~ns}$ run (Figure 3). Overall, the number of doxorubicin molecules in close proximity to the silk fibroin model was higher at $\mathrm{pH} 7.4$ than that at $\mathrm{pH}$ 4. This indicated that doxorubicin had a higher binding affinity for silk fibroin at $\mathrm{pH} 7.4$ than that at an acidic $\mathrm{pH}$. Interestingly, the $\mathrm{N}$ terminus-doxorubicin system showed a significantly higher number of doxorubicin molecules at a long distance from the silk structure $(>2 \mathrm{~nm})$ at $\mathrm{pH} 4$ than that at the neural $\mathrm{pH}$. This result suggests that the $\mathrm{N}$-terminal domain is an important structural element that influences doxorubicin release.

The intermolecular hydrogen bonds formed between doxorubicin and each silk fibroin structure model were calculated because the number of hydrogen bonds between the drug and the carriers is a key feature that modulates drug loading, according to previous research. ${ }^{38}$ The intermolecular hydrogen bond cutoff distance was set at $0.35 \mathrm{~nm}$ with a $30^{\circ}$ cutoff angle. ${ }^{39}$ Overall, the number of intermolecular hydrogen bond systems was higher in the $\mathrm{pH} 7.4$ systems than that in the $\mathrm{pH} 4$ systems, which indicated a high drug loading efficacy at $\mathrm{pH} 7.4$ (Figure 3). The number of hydrogen bonds between doxorubicin and the $\mathrm{N}$-terminal domain of silk fibroin was also substantially higher at $\mathrm{pH} 7.4$ than that at $\mathrm{pH} 4$ (Figure 3). Therefore, this result proved that the $\mathrm{N}$-terminal domain had a substantial influence on the drug loading and drug release behavior.

The interaction energies were calculated to determine the binding strength and the types of intermolecular interactions between doxorubicin and silk fibroin. The three prominent intermolecular forces can be classified in the following rank order: electrostatic interaction $>$ hydrogen bonding $>$ van der Waals interactions. ${ }^{40}$ Overall, the total interaction energies were higher for the $\mathrm{pH} 7.4$ systems than those for the $\mathrm{pH} 4$ systems, indicating a greater doxorubicin-silk interaction at pH 7.4 (Figure S6). The total energies were also higher in the crystalline silk structure (Figure S6A) than those in the amorphous silk structure (Figure S6C), indicating a lower doxorubicin release from the crystalline structure; this in silico prediction is in agreement with experimental data sets. ${ }^{7,8}$ Electrostatic interactions were also important at $\mathrm{pH} 7.4$, especially for the N-terminal-doxorubicin pair (Figure S6D), due to the high number of hydrogen bonds (>20) (Figure 3). Therefore, the electrostatic interaction energy and the number of hydrogen bonds were plotted to determine the relationship between these values. The number of hydrogen bonds was strongly correlated with the electrostatic interaction between silk fibroin and doxorubicin, and it correlated well with previous work on doxorubicin-chitosan oligosaccharide interactions. ${ }^{17}$ An increasing electrostatic interaction energy led to a greater number of hydrogen bonds (Figure S7).

LIGPLOT and Discovery Studio were then used to generate a $2 \mathrm{D}$ interaction diagram and to monitor which amino acids of silk fibroin interacted with doxorubicin at 50 ns. The $2 \mathrm{D}$ diagram of the LIGPLOT detailed hydrogen bonds (green lines) and hydrophobic interactions (gray arcs) between silk 
fibroin and doxorubicin (Figure S8). The doxorubicin molecule in LIGPLOT was selected from those that showed the highest interaction energies with the silk fibroin model at $50 \mathrm{~ns}$. Overall, the number of hydrogen bonds and the number of amino acids responsible for the silk fibroin doxorubicin interaction were higher at $\mathrm{pH} 7.4$ than those at $\mathrm{pH} 4$ (Figure S8 and Table S5). For the silk fibroin structure with the highest $\beta$-sheet content, the interaction between silk and doxorubicin at $\mathrm{pH} 4$ was dominated by hydrophobic interactions (Figure S8A), while the interaction at $\mathrm{pH} 7.4$ was dominated by hydrogen bonds. For other silkdoxorubicin model systems, the two-dimensional diagram from LIGPLOT did not show significant differences in response to $\mathrm{pH}$ changes.

Overall, $\mathrm{pH}$ influenced the doxorubicin binding affinity toward silk fibroin because the ionizable amino acid residues (especially glutamic and aspartic acid) on the fibroin structure provided a greater number of negative charges at $\mathrm{pH} 7.4$ than they did at $\mathrm{pH} 4$ (shown in red in Table S5). This correlates well with previous wet-lab experiments showing $\mathrm{pH}$-dependent drug release $4,7,8,11,36$ and indicates that modulation of the numbers of ionizable groups could be a useful approach for further refinement of the design of chemically-modified or recombinant silks intended for drug delivery. ${ }^{14}$

In summary, the well-tempered metadynamics simulation enabled us to explore $>1000$ amorphous-crystalline silk fibroin structures and yielded several useful silk conformations, including both previously reported and novel silk structures (all of these structures are now freely available to download). The molecular interaction between these silk fibroin structures and doxorubicin showed $\mathrm{pH}$-dependent effects. The $\mathrm{N}$ terminal domain was proven to be an important "switch" that controls drug binding and release in response to $\mathrm{pH}$. This $\mathrm{pH}$ control was attributed to ionizable amino acid residues, especially glutamic acid. The electrostatic interactions due to hydrogen bonds were of critical importance for drug loading and release. Overall, this study demonstrated important structure-function relationships that will help in the development of recombinant silks with improved drug binding and release characteristics.

\section{ASSOCIATED CONTENT}

\section{S Supporting Information}

The Supporting Information is available free of charge on the ACS Publications website at DOI: 10.1021/acs.jpclett.9b01591.

Access to the full data set, time evolution of the collective variables and system free energy, time evolution of the free energy landscapes, Ramachandran plot for the repetitive sequence motifs, calculated $\mathrm{p} K_{\mathrm{a}}$ from Discovery Studio and ionizable side chain of amino acids, surface charge distributions, interaction energies between doxorubicin and the model silks, linear relationship between the number of hydrogen bonds and electrostatic interaction energy of the silkdoxorubicin system, schematic representation of the two-dimensional doxorubicin structure with the $\mathrm{H}$ bonds and hydrophobic interactions between the amino acid residues of the silk fibroin structure, calculated $\mathrm{pK}_{\mathrm{a}}$ values of ionizable amino acids under various conditions, and list of amino acid residues in each model silk structure (PDF)

\section{AUTHOR INFORMATION}

\section{Corresponding Authors}

*E-mail: blair.johnston@strath.ac.uk. Tel. +44 (0) 141548 5756 (B.F.J.).

*E-mail: philipp.seib@strath.ac.uk. Tel. +44 (0) 1415482510 (F.P.S.).

ORCID

Antony D. Vassileiou: 0000-0001-8146-8972

Samir H. Mushrif: 0000-0002-0002-9634

Blair F. Johnston: 0000-0001-9785-6822

F. Philipp Seib: 0000-0002-1955-1975

\section{Author Contributions}

T.W. designed, analyzed, and interpreted the data and generated the manuscript draft. A.D.V. contributed to the interpretation of the results. All authors (T.W., A.D.V., S.G., S.H.M., B.F.J., and F.P.S.) discussed the results and/or provided advice on the experimental design and analysis. B.F.J. and F.P.S. conceived the study and supervised the project. F.P.S. content edited the manuscript with support from the other authors.

\section{Notes}

The authors declare no competing financial interest.

\section{ACKNOWLEDGMENTS}

The authors thank the International Strategic Partnership between the University of Strathclyde and Nanyang Technological University, Singapore for funding this work. The authors acknowledge that this work was carried out in part at the EPSRC Future Manufacturing Research Hub for Continuous Manufacturing and Advanced Crystallization (CMAC) (EP/P006965/1), supported by a U.K. Research Partnership Fund award from the Higher Education Funding Council for England (Grant HH13054).

\section{REFERENCES}

(1) Omenetto, F. G.; Kaplan, D. L. New Opportunities for an Ancient Material. Science 2010, 329 (5991), 528-531.

(2) Holland, C.; Numata, K.; Rnjak-Kovacina, J.; Seib, F. P. The Biomedical Use of Silk: Past, Present, Future. Adv. Healthcare Mater. 2019, 8, 1800465.

(3) Altman, G. H.; Diaz, F.; Jakuba, C.; Calabro, T.; Horan, R. L.; Chen, J.; Lu, H.; Richmond, J.; Kaplan, D. L. Silk-Based Biomaterials. Biomaterials 2003, 24 (3), 401-416.

(4) Totten, J. D.; Wongpinyochit, T.; Seib, F. P. Silk Nanoparticles: Proof of Lysosomotropic Anticancer Drug Delivery at Single-Cell Resolution. J. Drug Target. 2017, 25 (9-10), 865-872.

(5) Yucel, T.; Lovett, M. L.; Kaplan, D. L. Silk-Based Biomaterials for Sustained Drug Delivery. J. Controlled Release 2014, 190, 381397.

(6) Zhao, Z.; Li, Y.; Xie, M.-B. Silk Fibroin-Based Nanoparticles for Drug Delivery. Int. J. Mol. Sci. 2015, 16 (3), 4880-4903.

(7) Seib, F. P.; Kaplan, D. L. Doxorubicin-Loaded Silk Films: DrugSilk Interactions and in Vivo Performance in Human Orthotopic Breast Cancer. Biomaterials 2012, 33 (33), 8442-8450.

(8) Coburn, J. M.; Na, E.; Kaplan, D. L. Modulation of Vincristine and Doxorubicin Binding and Release from Silk Films. J. Controlled Release 2015, 220, 229-238.

(9) Seib, F. P.; Pritchard, E. M.; Kaplan, D. L. Self-Assembling Doxorubicin Silk Hydrogels for the Focal Treatment of Primary Breast Cancer. Adv. Funct. Mater. 2013, 23 (1), 58-65.

(10) Poursaid, A.; Jensen, M. M.; Nourbakhsh, I.; Weisenberger, M.; Hellgeth, J. W.; Sampath, S.; Cappello, J.; Ghandehari, H. SilkElastinlike Protein Polymer Liquid Chemoembolic for Localized 
Release of Doxorubicin and Sorafenib. Mol. Pharmaceutics 2016, 13, 2736-2748.

(11) Wongpinyochit, T.; Uhlmann, P.; Urquhart, A. J.; Seib, F. P. PEGylated Silk Nanoparticles for Anticancer Drug Delivery. Biomacromolecules 2015, 16 (11), 3712-3722.

(12) Xia, X.-X.; Wang, M.; Lin, Y.; Xu, Q.; Kaplan, D. L. Hydrophobic Drug-Triggered Self-Assembly of Nanoparticles from Silk-Elastin-like Protein Polymers for Drug Delivery. Biomacromolecules 2014, 15 (3), 908-914.

(13) Harris, J.; Klonoski, S. C.; Chiu, B. Clinical Considerations of Focal Drug Delivery in Cancer Treatment. Curr. Drug Delivery 2017, 14 (5), 588-596.

(14) Kucharczyk, K.; Weiss, M.; Jastrzebska, K.; Luczak, M.; Ptak, A.; Kozak, M.; Mackiewicz, A.; Dams-Kozlowska, H. Bioengineering the Spider Silk Sequence to Modify Its Affinity for Drugs. Int. J. Nanomed. 2018, 13, 4247-4261.

(15) Adnan, A.; Lam, R.; Chen, H.; Lee, J.; Schaffer, D. J.; Barnard, A. S.; Schatz, G. C.; Ho, D.; Liu, W. K. Atomistic Simulation and Measurement of $\mathrm{pH}$ Dependent Cancer Therapeutic Interactions with Nanodiamond Carrier. Mol. Pharmaceutics 2011, 8 (2), 368-374.

(16) Mahdavi, M.; Rahmani, F.; Nouranian, S. Molecular Simulation of $\mathrm{pH}$-Dependent Diffusion, Loading, and Release of Doxorubicin in Graphene and Graphene Oxide Drug Delivery Systems. J. Mater. Chem. B 2016, 4 (46), 7441-7451.

(17) Shan, P.; Shen, J.-W.; Xu, D.-H.; Shi, L.-Y.; Gao, J.; Lan, Y.-W.; Wang, Q.; Wei, X.-H. Molecular Dynamics Study on the Interaction between Doxorubicin and Hydrophobically Modified Chitosan Oligosaccharide. RSC Adv. 2014, 4 (45), 23730.

(18) Beck, D. A. C.; Daggett, V. Methods for Molecular Dynamics Simulations of Protein Folding/unfolding in Solution. Methods 2004, 34 (1), 112-120.

(19) Arinaminpathy, Y.; Sansom, M. S. P.; Biggin, P. C. Molecular Dynamics Simulations of the Ligand-Binding Domain of the Ionotropic Glutamate Receptor GluR2. Biophys. J. 2002, 82 (2), 676-683.

(20) Keten, S.; Xu, Z.; Ihle, B.; Buehler, M. J. Nanoconfinement Controls Stiffness, Strength and Mechanical Toughness of $\beta$-Sheet Crystals in Silk. Nat. Mater. 2010, 9 (4), 359-367.

(21) Xiao, S.; Xiao, S.; Gräter, F. Dissecting the Structural Determinants for the Difference in Mechanical Stability of Silk and Amyloid Beta-Sheet Stacks. Phys. Chem. Chem. Phys. 2013, 15, 87658771.

(22) Cheng, Y.; Koh, L.; Li, D.; Ji, B.; Han, M.; Zhang, Y. On the Strength of $\beta$-Sheet Crystallites of Bombyx Mori Silk Fibroin. J. $R$. Soc., Interface 2014, 11, 20140305.

(23) Cheng, Y.; Koh, L.-D.; Li, D.; Ji, B.; Zhang, Y.; Yeo, J.; Guan, G.; Han, M.-Y.; Zhang, Y.-W. Peptide-Graphene Interactions Enhance the Mechanical Properties of Silk Fibroin. ACS Appl. Mater. Interfaces 2015, 7 (39), 21787-21796.

(24) Xiao, S.; Stacklies, W.; Cetinkaya, M.; Markert, B.; Gräter, F. Mechanical Response of Silk Crystalline Units from ForceDistribution Analysis. Biophys. J. 2009, 96 (10), 3997-4005.

(25) Zhao, B.; Cohen Stuart, M. A.; Hall, C. K. Navigating in Foldonia: Using Accelerated Molecular Dynamics to Explore Stability, Unfolding and Self-Healing of the $\beta$-Solenoid Structure Formed by a Silk-like Polypeptide. PLoS Comput. Biol. 2017, 13 (3), e1005446.

(26) Ha, S.-W.; Gracz, H. S.; Tonelli, A. E.; Hudson, S. M. Structural Study of Irregular Amino Acid Sequences in the Heavy Chain of Bombyx Mori Silk Fibroin. Biomacromolecules 2005, 6, 2563-2569.

(27) Durrant, J. D.; McCammon, J. A. Molecular Dynamics Simulations and Drug Discovery. BMC Biol. 2011, 9 (1), 71.

(28) Laio, A.; Gervasio, F. L. Metadynamics: A Method to Simulate Rare Events and Reconstruct the Free Energy in Biophysics, Chemistry and Material Science. Rep. Prog. Phys. 2008, 71, 126601.

(29) Barducci, A.; Bussi, G.; Parrinello, M. Well-Tempered Metadynamics: A Smoothly Converging and Tunable Free-Energy Method. Phys. Rev. Lett. 2008, 100 (2), 1-4.
(30) Dama, J. F.; Parrinello, M.; Voth, G. A. Well-Tempered Metadynamics Converges Asymptotically. Phys. Rev. Lett. 2014, 112 (24), 240602.

(31) He, Y. X.; Zhang, N. N.; Li, W. F.; Jia, N.; Chen, B. Y.; Zhou, K.; Zhang, J.; Chen, Y.; Zhou, C. Z. N-Terminal Domain of Bombyx Mori Fibroin Mediates the Assembly of Silk in Response to $\mathrm{pH}$ Decrease. J. Mol. Biol. 2012, 418, 197-207.

(32) Barducci, A.; Bonomi, M.; Parrinello, M. Metadynamics. Wiley Interdiscip. Rev. Comput. Mol. Sci. 2011, 1 (5), 826-843.

(33) Asakura, T.; Okushita, K.; Williamson, M. P. Analysis of the Structure of Bombyx Mori Silk Fibroin by NMR. Macromolecules 2015, 48, 2345-2357.

(34) Suzuki, Y.; Yamazaki, T.; Aoki, A.; Shindo, H.; Asakura, T. NMR Study of the Structures of Repeated Sequences, GAGXGA (X = S, Y, V), in Bombyx Mori Liquid Silk. Biomacromolecules 2014, 15, 104-112.

(35) Humphrey, W.; Dalke, A.; Schulten, K. VMD: Visual Molecular Dynamics. J. Mol. Graphics 1996, 14 (1), 33-38.

(36) Seib, F. P.; Jones, G. T.; Rnjak-Kovacina, J.; Lin, Y.; Kaplan, D. L. $\mathrm{pH}$-Dependent Anticancer Drug Release from Silk Nanoparticles. Adv. Healthcare Mater. 2013, 2 (12), 1606-1611.

(37) Pace, C. N.; Grimsley, G. R.; Scholtz, J. M. Protein Ionizable Groups: pK Values and Their Contribution to Protein Stability and Solubility. J. Biol. Chem. 2009, 284 (20), 13285-13289.

(38) Subashini, M.; Devarajan, P. V.; Sonavane, G. S.; Doble, M. Molecular Dynamics Simulation of Drug Uptake by Polymer. J. Mol. Model. 2011, 17 (5), 1141-1147.

(39) Van Der Spoel, D.; Lindahl, E.; Hess, B.; Groenhof, G.; Mark, A. E.; Berendsen, H. J. C. GROMACS: Fast, Flexible, and Free. J. Comput. Chem. 2005, 26 (16), 1701-1718.

(40) Dhotel, A.; Chen, Z.; Delbreilh, L.; Youssef, B.; Saiter, J.-M.; Tan, L. Molecular Motions in Functional Self-Assembled Nanostructures. Int. J. Mol. Sci. 2013, 14 (2), 2303-2333. 